\title{
Pirfenidone inhibits fibrosis in foreign body reaction after glaucoma drainage device implantation
}

This article was published in the following Dove Press journal:

Drug Design, Development and Therapy

I5 April 2016

Number of times this article has been viewed

\author{
Kyoung In Jung \\ Chan Kee Park \\ Department of Ophthalmology \\ and Visual Science, Seoul St Mary's \\ Hospital, College of Medicine, The \\ Catholic University of Korea, Seoul, \\ South Korea
}

Background: The aim of this study was to investigate the antiscarring effects of pirfenidone on foreign body reaction in a rabbit model of glaucoma drainage implant surgery.

Methods: Adult New Zealand White rabbits had glaucoma drainage device implantation using Model FP8 Ahmed glaucoma valves. One eye was randomly assigned to receive postoperative intrableb injection of pirfenidone followed by topical treatment. The other eye underwent the same procedure but without the addition of pirfenidone. Histochemical staining and immunohistochemistry for blebs were performed.

Results: The degree of cellularity was smaller in the pirfenidone group than in the control group at 2 weeks post operation $(P=0.005)$. A few foreign body giant cells were detected in the inner border of the capsule, and their numbers were similar in the control and pirfenidone groups $(P>0.05)$. Using Masson's trichrome stain, the inner collagen-rich layer was found to be thinner in the pirfenidone group than the control group at 4 weeks $(P=0.031)$ and 8 weeks $(P=0.022)$ post operation. The percentage of proliferating cell nuclear antigen-positive cells was lower in the pirfenidone group than in the control group at 2 weeks post operation (total bleb, $P=0.022$; inner bleb, $P=0.036$ ). Pirfenidone treatment decreased the immunoreactivity of connective tissue growth factor at 2 weeks post operation (total bleb, $P=0.029$; inner bleb, $P=0.018$ ). The height and area of $\alpha$-smooth muscle actin expression were lower in the pirfenidone group than the control group at 2 weeks, 4 weeks, and 8 weeks post operation (all $P<0.05$ ).

Conclusion: Postoperative intrableb injection of pirfenidone followed by topical administration reduced fibrosis following glaucoma drainage device implantation. These findings suggest that pirfenidone may function as an antiscarring treatment in foreign body reaction after tube-shunt surgery.

Keywords: fibrosis, bleb, glaucoma drainage device surgery, transforming growth factor- $\beta$

\section{Introduction}

Glaucoma remains the main cause of irreversible visual loss globally. ${ }^{1}$ Glaucoma drainage device (GDD) implantation is preferred over trabeculectomy in patients with glaucoma with a high probability of failure. An increase in the popularity of GDD surgery to manage medically uncontrolled glaucoma has been reported. ${ }^{2-4}$ GDD surgery has shown a higher success rate with a lower incidence of postoperative complications than trabeculectomy. ${ }^{5}$ However, even in tube surgery, the failure rate was $33 \%$ at 5-year follow-up. ${ }^{5}$ If the primary GDD fails, additional tube surgery is more challenging. ${ }^{6,7}$ Therefore, success after the first tube surgery is critical for the treatment of refractory glaucoma.
Correspondence: Chan Kee Park Department of Ophthalmology and Visual Science, Seoul St Mary's Hospital, College of Medicine, The Catholic University of Korea, 505 Banpo-dong, Seocho-ku, Seoul I37-70I, South Korea

$\mathrm{Tel}+8225906199$

Fax +82 25997405

Email ckpark@catholic.ac.kr
Drug Design, Development and Therapy 2016:10 1477-|488

(c) (1) (8) ๑ 2016 Jung and Park. This work is published and licensed by Dove Medical Press Limited. The full terms of this license are available at https:/www.dovepress.com/terms.php cc. hereby accept the Terms. Non-commercial uses of the work are permitted without any further permission from Dove Medical Press Limited, provided the work is properly attributed. For permission for commercial use of this work, please see paragraphs 4.2 and 5 of our Terms (https://www.doveppess. com/terms.php).
Dovepress

http://dx.doi.org/1 0.2147/DDDT.S99957

\section{7}

.


Following seton glaucoma surgery, it is the collagen-rich capsule that poses the major resistance to aqueous humor flow through GDD. The postoperative intraocular pressure (IOP) seems to be poorly controlled with a thicker bleb and a lower total surface area. ${ }^{7-9}$

Wound-healing response may differ from that following trabeculectomy because a foreign body is inserted..$^{10}$ Histopathologically, foreign body reaction was found in the blebs, which is characterized by a collagen-rich capsule with a few foreign body giant cells (FBGCs). ${ }^{10}$ Previously, we reported that the aqueous humor increased fibrosis in the bleb after Ahmed valve implantation in rabbits. ${ }^{10}$ Aqueous humor contains a "cocktail" of different growth factors such as the transforming growth factor- $\beta$ (TGF- $\beta$ ), the basic fibroblast growth factor, epidermal growth factor, insulin-like growth factor-1 (IGF-1), and platelet-derived growth factor. ${ }^{11}$ Several growth factors involving TGF- $\beta$ contribute to the woundhealing response as chemotactic or mitogenic factors for fibroblasts. ${ }^{12}$ Therefore, the growth factors may be potent targets to decrease fibrosis in GDD surgery.

Pirfenidone (5-methyl-1-phenyl-2[1H]-pyridone), a modified derivative of pyridine, is one of the main treatment options for idiopathic pulmonary fibrosis. ${ }^{13-16}$ A growing body of evidence has shown that it has antifibrotic properties in many fibrotic disorders including idiopathic pulmonary fibrosis, although its precise molecular mechanisms have not yet been elucidated. ${ }^{13-18}$ Suppression of connective tissue growth factor (CTGF), ${ }^{19}$ platelet-derived growth factor, ${ }^{20}$ $\alpha$-smooth muscle actin ( $\alpha$-SMA), ${ }^{19}$ and TGF- $\beta^{14}$ is induced by the application of pirfenidone. In an in vitro study, pirfenidone was found to suppress proliferation and collagen contraction of human tenon's fibroblasts. ${ }^{21}$

Therefore, we hypothesized that pirfenidone might effectively reduce fibrosis of the capsule surrounding the GDD endplate. To our knowledge, there has been no report with regard to the effects of pirfenidone on foreign body reaction. In this study, we investigated whether pirfenidone use as an antiscarring agent affected the foreign body reaction after GDD implantation in a rabbit model.

\section{Methods}

\section{Animals}

Adult New Zealand White rabbits (2.0-3.0 kg; 20 weeks old) were used in accordance with the Association for Research in Vision and Ophthalmology (ARVO) Statement for the Use of Animals in Ophthalmic and Vision Research. All procedures for this study were approved by the Institutional Animal Care and Use Committee of the School of Medicine,
Catholic University of Korea (CUMC-2014-0142). Five animals were used in each time period (nonoperated normal control, 2 weeks, 4 weeks, and 8 weeks post operation). The total number of animals used was 20 .

\section{Glaucoma aqueous shunt model}

GDD implantations were performed using Model FP8 Ahmed glaucoma valves (New World Medical Inc., Rancho Cucamonga, CA, USA). The details of this technology for implantation of GDD have been described previously. ${ }^{10}$ Ahmed glaucoma valves were implanted in both eyes of the animals; the right or left eye was randomly allocated to the pirfenidone group and the other eye to the control group.

The operation was performed under general anesthesia by intramuscular injection of $15 \mathrm{mg} / \mathrm{kg}$ tiletamine plus zolazepam (Zoletil; Virbac, Carros, France) and $5 \mathrm{mg} / \mathrm{kg}$ xylazine hydrochloride (Rompun; Bayer, Leverkusen, Germany). Local anesthesia was achieved using proparacaine hydrochloride (Alcaine 0.5\%; Alcon, Fort Worth, TX, USA).

A fornix-based conjunctival flap was made in the temporal side of the superior quadrant. The plate was implanted under the conjunctiva and Tenon's capsule and was fixed to the sclera. Priming of the GDD was done by irrigation with saline through the tube, confirming patency before insertion. The tube was cut to allow its extension $2 \mathrm{~mm}$ to $3 \mathrm{~mm}$ into the anterior chamber (AC). The AC was entered through limbal incision with a $23 \mathrm{G}$ needle. A mixture of sodium hyaluronate $1.65 \%$ and sodium chondroitin sulfate $4 \%$ was injected to reform the $\mathrm{AC}$, which was followed by the insertion of tube into the AC. The tube was then loosely fixed to the sclera with a nonabsorbable suture. Reapproximation of the conjunctiva was done with 8-0 Vicryl sutures.

\section{Drug treatment}

Pirfenidone (P2116; Sigma-Aldrich, St Louis, MO, USA) was dissolved in carboxymethylcellulose (CMC) 0.5\% (Refresh Plus; Allergan, Inc., Irvine, CA, USA). Immediately after implantation of the Ahmed valve, an intrableb injection of pirfenidone $(1 \mathrm{mg} / \mathrm{mL}, 200 \mu \mathrm{L})$ was administered, followed by topical application of pirfenidone ( $5 \mathrm{mg} / \mathrm{mL}$, one drop) three times a day until the rabbit was sacrificed. The other eye, assigned to the control group, had an intrableb injection $(200 \mu \mathrm{L})$ of CMC $0.5 \%$, followed by topical application of $\mathrm{CMC}$ (one drop) three times a day.

\section{Measurement of IOP}

Measurement of IOP was performed under general anesthesia at 2 weeks, 4 weeks, and 8 weeks post operation. IOP was 
measured using a rebound tonometer $\left(\right.$ TonoVet $\left.^{\mathbb{}}\right)$. Five measurements of IOP were averaged.

\section{Gross examination of blebs after tube implantation}

At 2 weeks, 4 weeks, and 8 weeks post operation, five randomly selected animals were sacrificed by intravenous administration of potassium chloride. Before enucleation, all eyes underwent slit-lamp examination to confirm the patent tube tip and to evaluate the bleb appearance. After all eyes had been enucleated, they were placed overnight in $4 \%$ paraformaldehyde at room temperature.

\section{Histochemical staining}

After fixation, the bleb was cut over the endplate along the line of the tube for histological examination (Figure 1A). The sections on both sides along the midline were used in this study (Figure 1B). The tissues from the total central portion of the blebs were embedded in paraffin. Ten sections per eye were produced to a thickness of $3 \mu \mathrm{m}$. They were stained using hematoxylin and eosin (H\&E) or Masson's trichrome staining kit (HT15; Sigma-Aldrich) to identify collagen.

\section{Immunohistochemistry}

Immunohistochemical staining was performed using standard protocols with dextran polymer reagents (Envision + DAM system; Dako Denmark A/S, Glostrup, Denmark). For antigen retrieval, sections were placed for 20 minutes at $95^{\circ} \mathrm{C}$ in Target Retrieval Solution, citrate pH 6 (Dako Denmark A/S) using PTLink (Dako Denmark A/S). The slides were immersed in peroxidase blocking solution (Dako Denmark A/S) for
10 minutes and then with antiproliferating cell nuclear antigen (PCNA; 1:500; Sigma-Aldrich), monoclonal anti $\alpha$-SMA (1:4,500; Sigma-Aldrich), and CTGF (1:100; Santa Cruz Biotechnology Inc., Dallas, TX, USA). Antibodies were detected using the dextran polymer reagent for mouse antibody for 30 minutes. Slides were stained with 3,3'-diaminobenzidine for 10 minutes, and the nuclei were stained with Meyer's hematoxylin for 2 minutes. Sections were mounted onto slides (Permount; Thermo Fisher Scientific, Waltham, MA, USA).

\section{Image analysis}

The slides were observed using a microscope (Nikon Corporation, Tokyo, Japan) and scanned through a panoramic MIDI scanner (3DHISTECH Ltd., Budapest, Hungary). The images produced were analyzed by built-in image analysis algorithms. In $\mathrm{H} \& \mathrm{E}$ staining, cellularity (number $/ \mathrm{mm}^{2}$ ) was measured as the number of cells divided by the total bleb area. The total bleb area was defined as the whole fibrous capsule including conjunctiva above the space where the GDD plate had been placed. The thickness of the collagen-rich innermost layer in the bleb was measured using the built-in distancemeasuring tool in the device. Five height readings along the capsule were averaged and compared between the two groups. Additionally, the blue (collagen)-stained area above the designated threshold was measured using the HistoQuant module in the panoramic MIDI scanner. The measurement of the blue-stained area was done in the total bleb area. To evaluate the density of collagen staining, the blue-stained area was divided by total bleb area.

The mean of five height values and the total area of the $\alpha$-SMA immunostained bleb were measured using the built-in tool in the scanner. The percentage of PCNA-positive

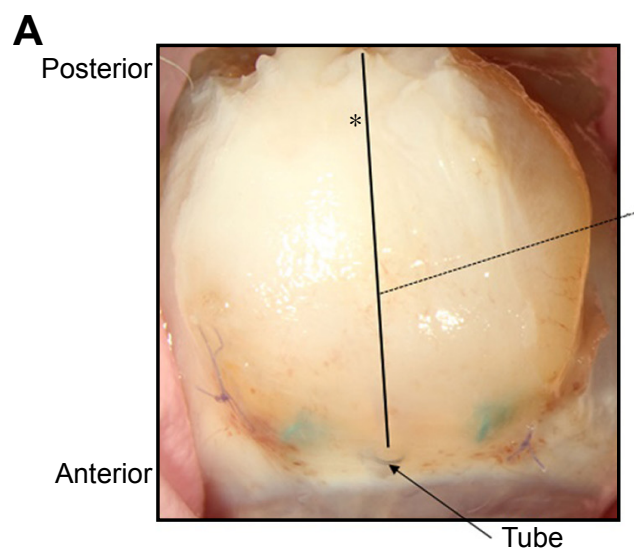

\section{B}

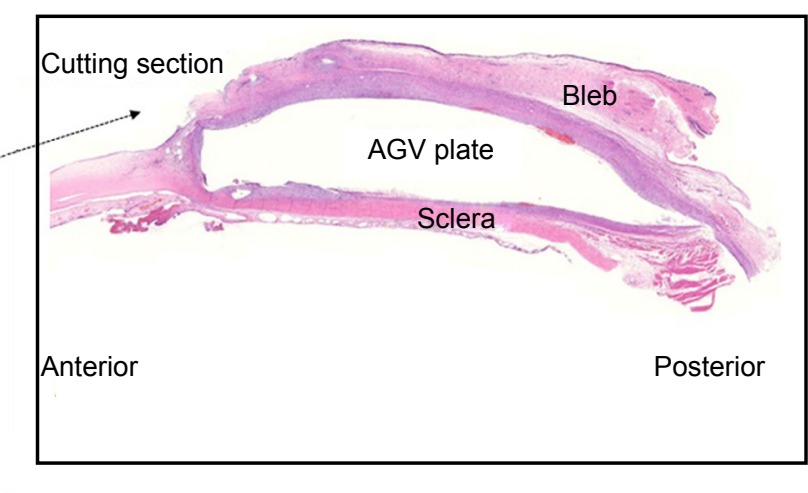

Figure I Preparation of the tissue for the bleb analysis.

Notes: (A) The bleb was cut above the plate along the midline (asterisk) corresponding to the axis of the tube. (B) The cross section just in the middle portion of the bleb was analyzed. The sections on both sides along the midline were used for the analysis.

Abbreviation: AGV, Ahmed glaucoma valve. 
cells and the CTGF-positive area in the total bleb area or the inner bleb area were calculated automatically using the HistoQuant module in the device.

\section{Statistical analysis}

Statistical analyses were performed using the SPSS software, Version 18.0 (SPSS Inc., Chicago, IL, USA). Differences in parameters between the control and pirfenidone groups were analyzed using a paired $t$-test. Values of $P<0.05$ indicate statistical significance.

\section{Results}

\section{Intraocular pressure}

Preoperative mean IOP was $9.1 \mathrm{mmHg}$ in the control group and $8.8 \mathrm{mmHg}$ in the pirfenidone group $(P=0.281)$. The respective mean postoperative IOPs were $8.9 \mathrm{mmHg}$, $8.6 \mathrm{mmHg}$, and $8.9 \mathrm{mmHg}$ at 2 weeks, 4 weeks, and 8 weeks post operation in the control group. In the pirfenidone group, the respective mean IOPs were $8.2 \mathrm{mmHg}, 7.1 \mathrm{mmHg}$, and $7.5 \mathrm{mmHg}$ at 2 weeks, 4 weeks, and 8 weeks post operation. IOPs were slightly lower in the pirfenidone group than in the control group, but the differences were not statistically significant for any period (all $P>0.05$ ).

\section{Histochemical staining}

In H\&E stain, the bleb revealed mild infiltration of inflammatory cells and prominent fibroblasts in the early woundhealing period (Figure $2 \mathrm{C}-\mathrm{H}$ ). The cellular density seemed to be greater in the inner bleb than in the subconjunctival outer bleb area in both groups. Overall, cellularity was reduced from 2 weeks to 8 weeks post operation. The degree of cellularity was smaller in the pirfenidone group than in the control group at 2 weeks post operation $(P=0.005$; Figure $2 \mathrm{~B})$. A few FBGCs were found along the inner portion of the bleb (Figure 2I) and around the suture materials (Figure 2J) in both groups. After excluding the FBGCs from around the suture material, the number of FBGCs in blebs was similar in the control and pirfenidone groups ( $P>0.05$; Figure $2 \mathrm{~K})$.

In the nonoperated eye, Masson's trichrome stain showed loosely arranged tenon's tissue stained weakly and sclera stained intensely blue (Figure 3A). Masson's trichrome stained the subepithelial collagen moderately blue in the bleb at 4 weeks post operation in both the control and pirfenidone groups (Figure 3C and D). Blue-stained collagen was mixed with pink-stained cytoplasm in the inner bleb area. The intensity of the blue stain in the innermost layer increased at 8 weeks post operation in both control and pirfenidone groups (Figure $3 \mathrm{E}$ and $\mathrm{F}$ ). The average of five height readings for the inner bleb was compared between the two groups (Figure 3B). The inner collagen-rich layer was thinner in the pirfenidone group than in the control group at 4 weeks and 8 weeks post operation ( $P=0.031$ and $P=0.022$, respectively; Figure $3 \mathrm{G}$ ). The collagen-stained area was less in the pirfenidone group than in the control group at 8 weeks post operation $(P=0.027$; Figure $3 \mathrm{H})$. The pirfenidone group also showed a lower density of collagen deposition than the control group at 8 weeks post operation ( $P=0.026$; Figure $3 \mathrm{I})$.

\section{Immunohistochemistry \\ Proliferation of fibroblasts}

The virgin conjunctiva, Tenon's capsule, and sclera revealed no positive staining for anti-PCNA (Figure 4A). The percentage of PCNA-positive cells was lower in the pirfenidone group $(57.8 \% \pm 9.9 \%)$ than in the control group $(80.2 \% \pm 12.1 \%)$ in the total blebs at 2 weeks post operation $(P=0.022$; Figure 4B-E, J). Cell expression of PCNA decreased at 4 weeks compared to that at 2 weeks post operation in both groups. The fractions of PCNA-positive cells were similar in the control and pirfenidone groups at 4 weeks post operation $(P=0.820$; Figure $4 \mathrm{~F}-\mathrm{J})$. For inner blebs, the proportion of PCNA-expressing cells was less in the pirfenidone group than in the control group at 2 weeks post operation $(P=0.036$; Figure $4 \mathrm{~K})$.

\section{Connective tissue growth factor}

CTGF hardly stained the normal tenon's capsule in the nonoperated eye (Figure 5A). CTGF signals were found throughout the bleb, especially in the inner bleb area in both groups (Figure 5B-I). Pirfenidone treatment decreased CTGF expression compared to the control group at 2 weeks in the total bleb after GDD implantation ( $P=0.029$; Figure $5 \mathrm{~J}$ ). The CTGF-positive area decreased from 2 weeks to 4 weeks post operation, and the difference between the two groups was not statistically significant at 4 weeks post operation $(P=0.250$; Figure 5J). Pirfenidone treatment decreased CTGF expression compared to the control group 2 weeks post operation after GDD implantation also in the inner bleb $(P=0.018$, Figure 5K).

\section{Transformation of fibroblasts to myofibroblasts}

Expression of $\alpha$-SMA is a reliable marker of the myofibroblast phenotype. ${ }^{22}$ No $\alpha$-SMA immunostaining was visible in virgin conjunctiva and sclera (Figure 6A). Immunostaining of $\alpha$-SMA was greatest in the inner portion of the bleb from 2 weeks to 8 weeks post operation (Figure 6B-H). The height and area of $\alpha$-SMA-positive blebs were greatest at 2 weeks post operation, 


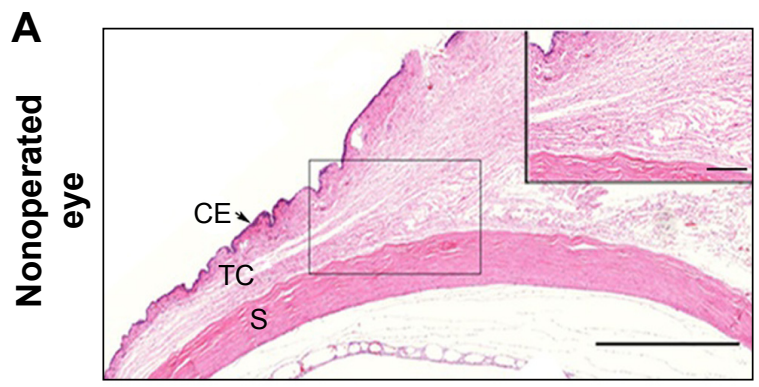

Control
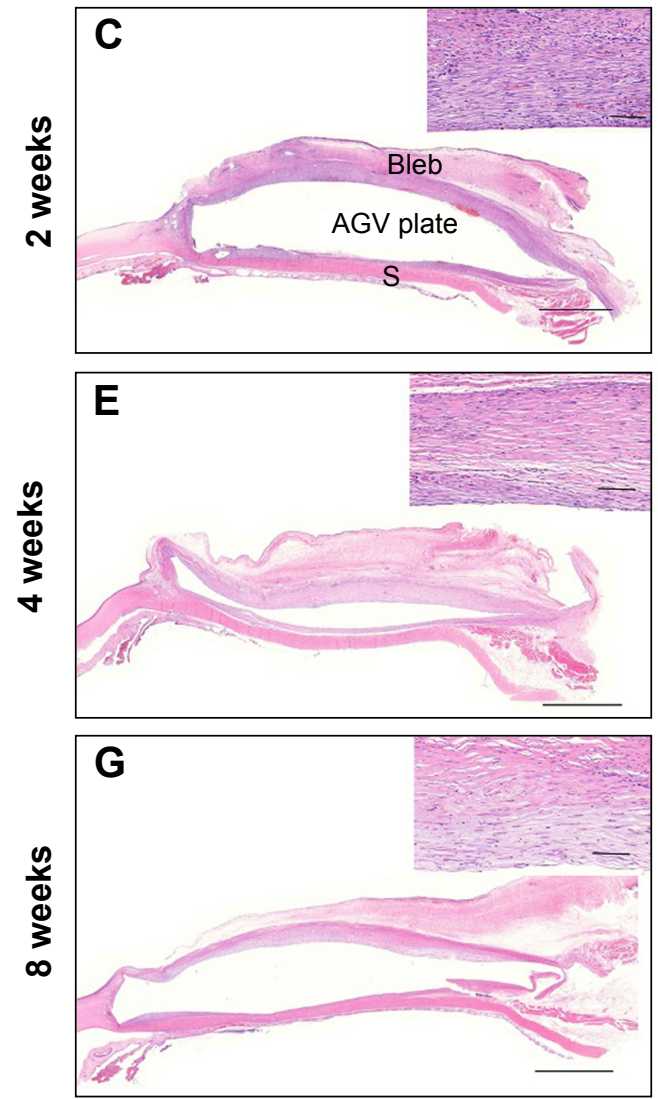

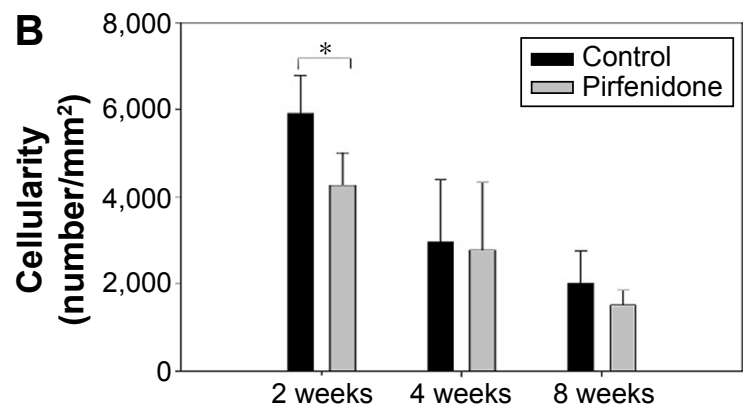

Pirfenidone
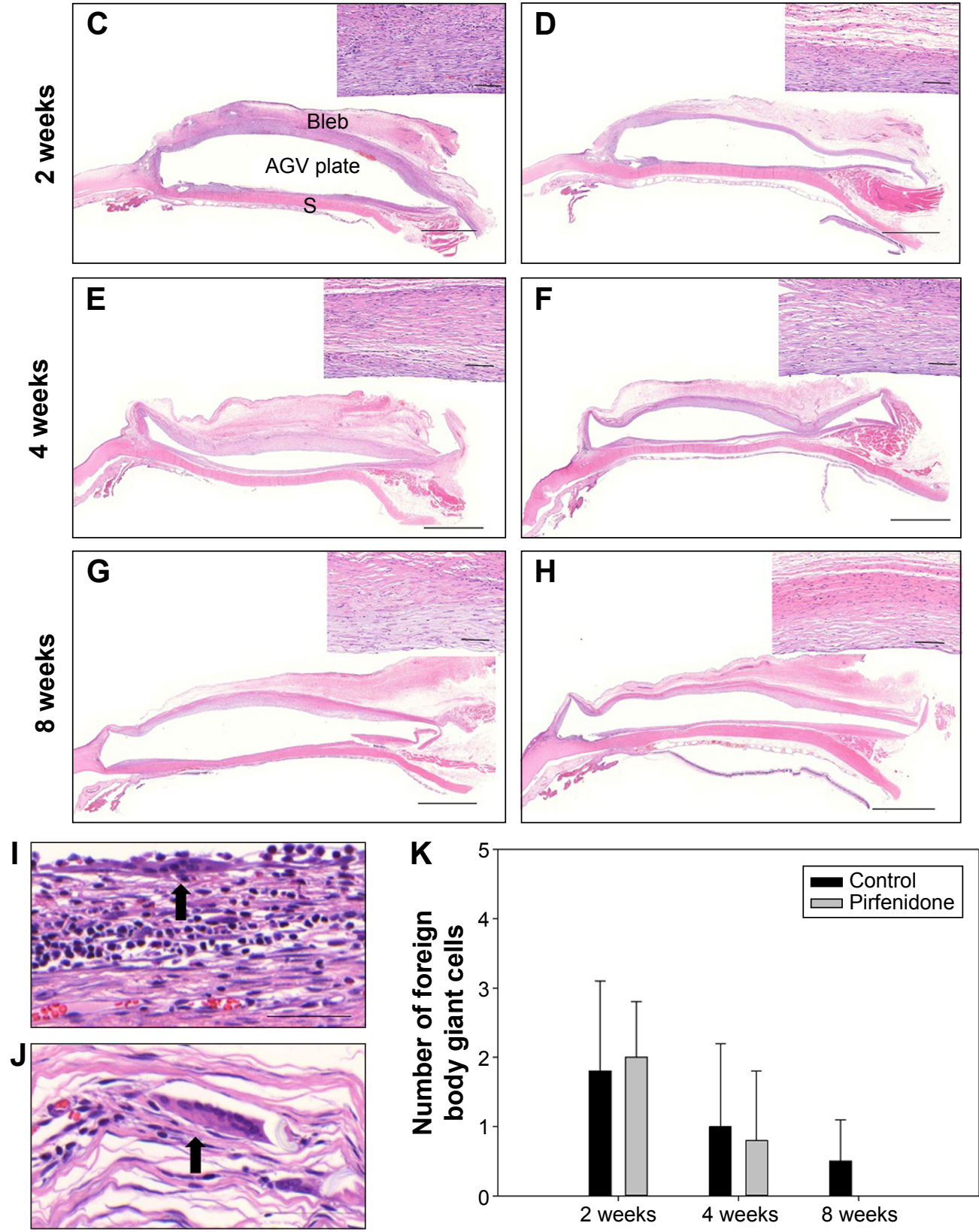

Figure 2 Histologic micrographs (H\&E staining) of bleb surrounding the endplate after glaucoma drainage device implantation.

Notes: (A) Virgin conjunctiva, Tenon's capsule, and sclera. The degree of cellularity in the total bleb was lower in the pirfenidone group than in the control group at 2 weeks post operation $(P=0.005, \mathbf{B})$. ( $\mathbf{C}-\mathbf{H})$ Bleb after glaucoma drainage device implantation. FBGCs (arrow) were observed in the inner bleb near the endplate at 4 weeks post operation (I) and around the suture material at 2 weeks after operation (J). The number of FBGCs was not different between the two groups (K). Scale bars: I,000 $\mu \mathrm{m}$, $200 \mu \mathrm{m}$ within the box $(\mathbf{A}) ; 2 \mathrm{~mm}(\mathbf{C}-\mathbf{H}), 100 \mu \mathrm{m}(\mathbf{C}-\mathbf{H}$, right upper part); $50 \mu \mathrm{m}(\mathbf{I}, \mathbf{J})$. *A significant difference with $P<0.05$ compared to control rats.

Abbreviations: AGV, Ahmed glaucoma valve; CE, conjunctival epithelium; FBGCs, foreign body giant cells; H\&E, hematoxylin and eosin; S, sclera; TC, Tenon's capsule. 

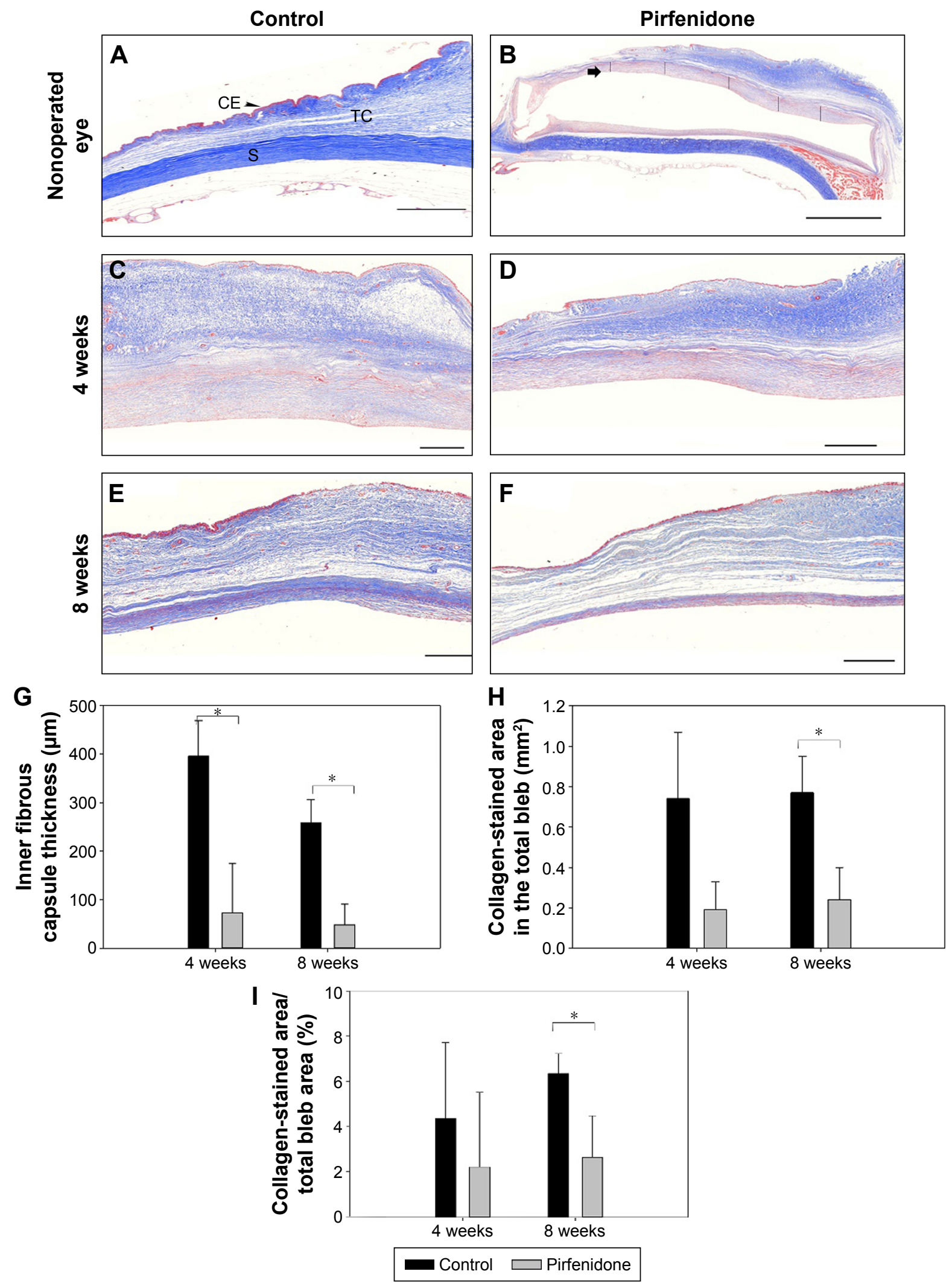

Figure 3 Bleb fibrosis represented by Masson's trichrome staining.

Notes: In the nonoperated eye, Masson's trichrome stain showed loosely arranged tenon's tissue stained weakly, and sclera stained intensely blue (A). The height (arrow) of the collagen-stained innermost layer in the bleb was measured, and the mean height was calculated from five height measurements (B). Pirfenidone treatment decreased the thickness of the inner fibrous layer at 4 weeks and 8 weeks post operation $(P=0.031$ and $P=0.022$, respectively; $\mathbf{C}-\mathbf{G})$. The collagen-stained area was less in the pirfenidone group than in the control group at 8 weeks post operation $(P=0.027 ; \mathbf{H})$. The pirfenidone group showed a lower density of collagen deposition than the control group at 8 weeks post operation $\left(P=0.026\right.$; I). Scale bars: $500 \mu \mathrm{m}(\mathbf{A}) ; 2 \mathrm{~mm}(\mathbf{B}) ; 500 \mu \mathrm{m}(\mathbf{C}-\mathbf{F})$. ${ }^{* A}$ significant difference with $P<0.05$ compared to control rats.

Abbreviations: $\mathrm{CE}$, conjunctival epithelium; S, sclera; TC, Tenon's capsule. 


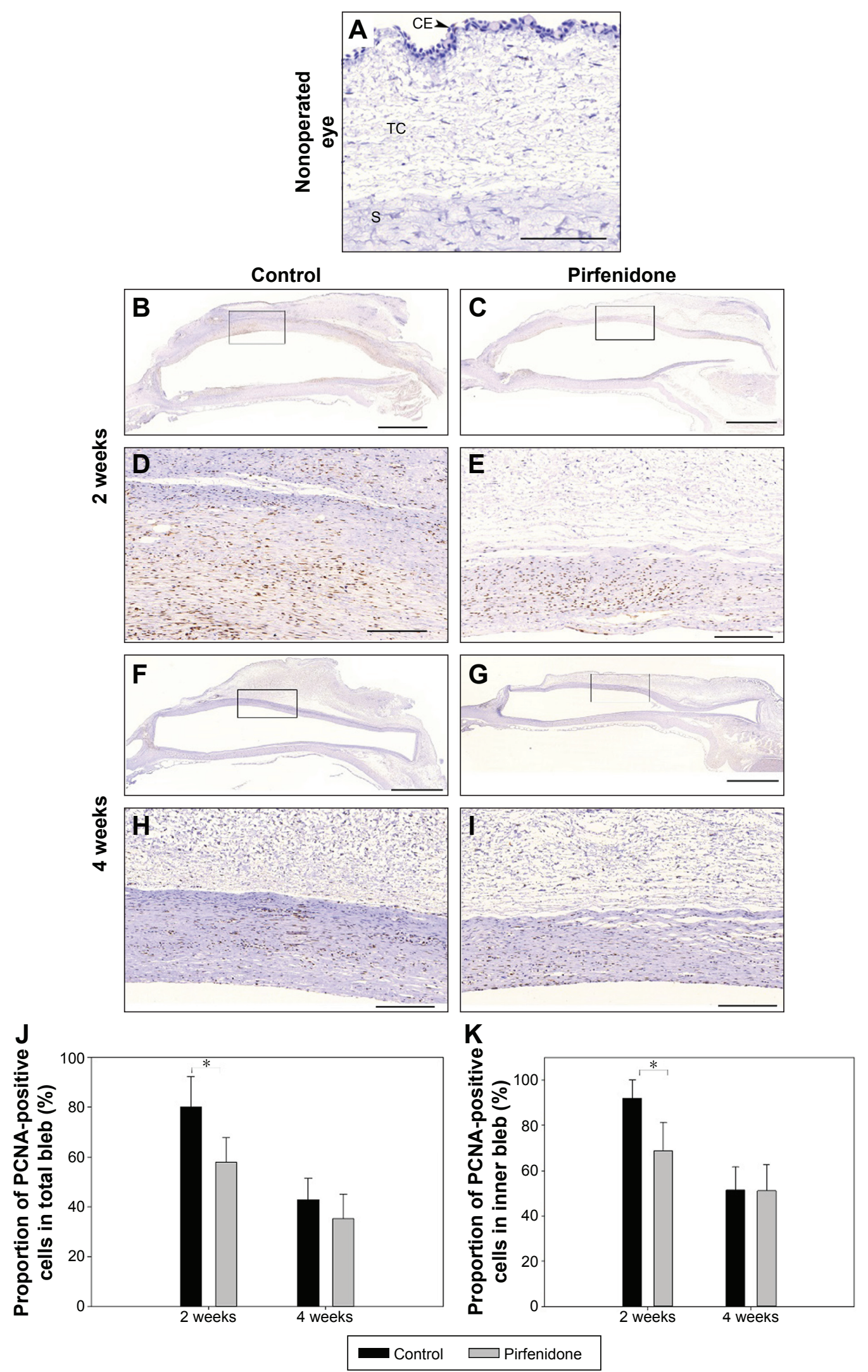

Figure 4 Immunohistochemical micrographs for cell division in the bleb.

Notes: Nonoperated conjunctiva, Tenon's capsule, and sclera revealed no positive staining for anti-PCNA (A). For total blebs, the percentage of PCNA-expressing cells was lower in the pirfenidone group than in the control group at 2 weeks post operation $(P=0.022 ; \mathbf{B}-\mathbf{E}$, J). At 4 weeks after operation, the difference between the two groups was not significant $(P=0.820 ; \mathbf{F}-\mathbf{J})$. For inner blebs, the proportion of $\mathrm{PCNA}$-expressing cells was less in the pirfenidone group than in the control group at 2 weeks post operation ( $P=0.036 ; \mathbf{K})$. Scale bars: $100 \mu \mathrm{m}(\mathbf{A}) ; 2 \mathrm{~mm}(\mathbf{B}, \mathbf{C}, \mathbf{F}, \mathbf{G}) ; 200 \mu \mathrm{m}(\mathbf{D}, \mathbf{E}, \mathbf{H}, \mathbf{I})$. ${ }^{*}$ A significant difference with $P<0.05$ compared to control rats.

Abbreviations: CE, conjunctival epithelium; PCNA, proliferating cell nuclear antigen; S, sclera; TC, Tenon's capsule. 


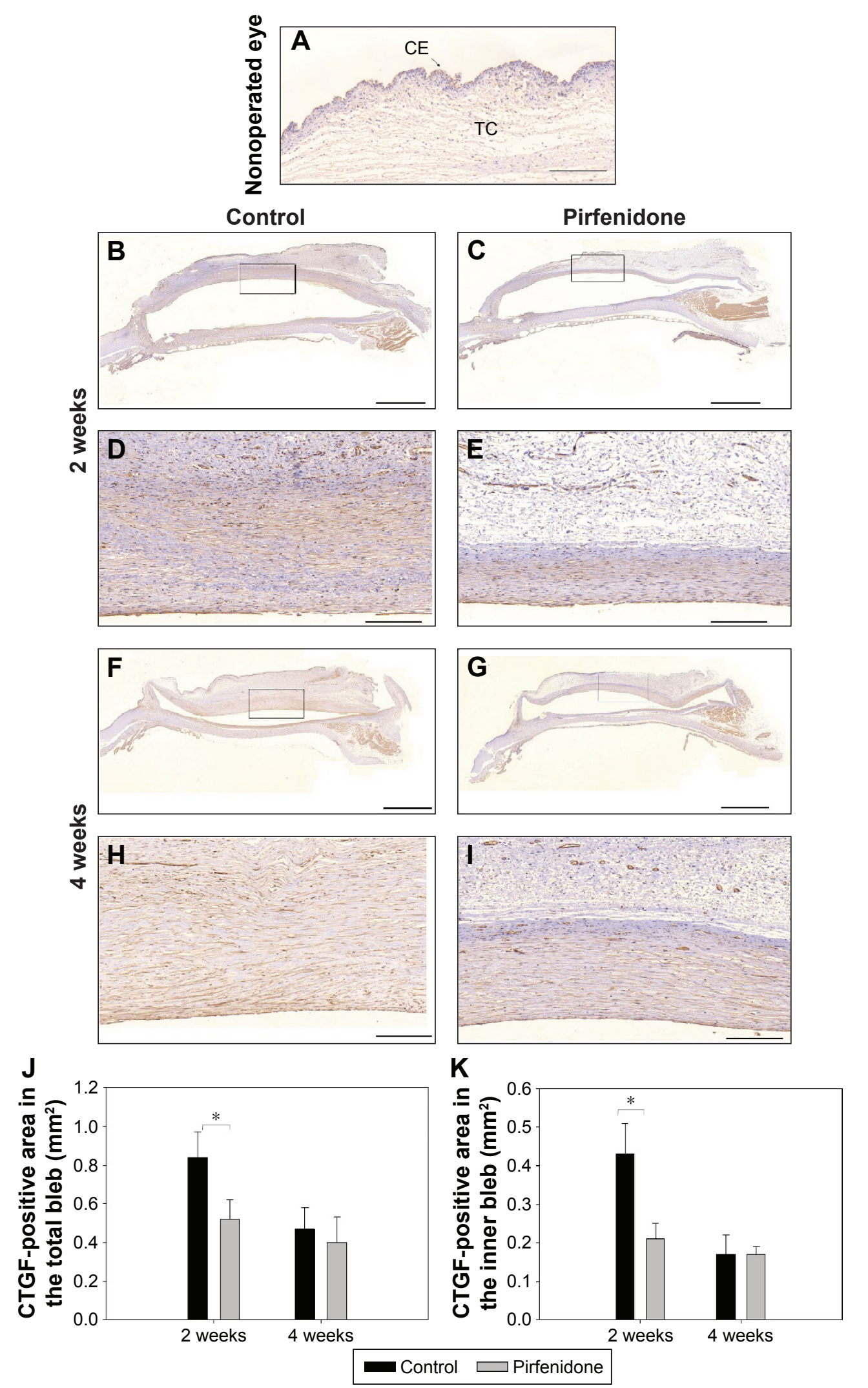

Figure 5 Immunohistochemical micrographs for CTGF.

Notes: CTGF scarcely stained the normal Tenon's capsule in the nonoperated eye (A). CTGF immunostaining was positive throughout the bleb, especially in the inner bleb area in both groups (B-I). Pirfenidone treatment decreased CTGF expression compared with the control group at 2 weeks after GDD implantation ( $P=0.029$, total bleb [J]; $P=0.018$, inner bleb [K]). Scale bars: $200 \mu \mathrm{m}(\mathbf{A}) ; 2 \mathrm{~mm}(\mathbf{B}, \mathbf{C}, \mathbf{F}, \mathbf{G}) ; 200 \mu \mathrm{m}(\mathbf{D}, \mathbf{E}, \mathbf{H}, \mathbf{I}) .{ }^{*}$ A significant difference with $P<0.05$ compared to control rats.

Abbreviations: CE, conjunctival epithelium; CTGF, connective tissue growth factor; GDD, glaucoma drainage device; TC, Tenon's capsule. 


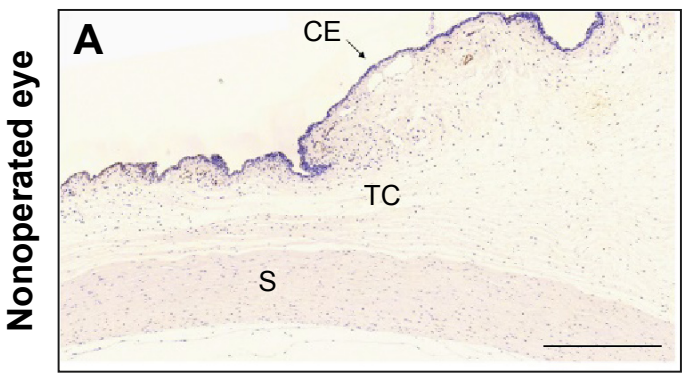

\section{Control}
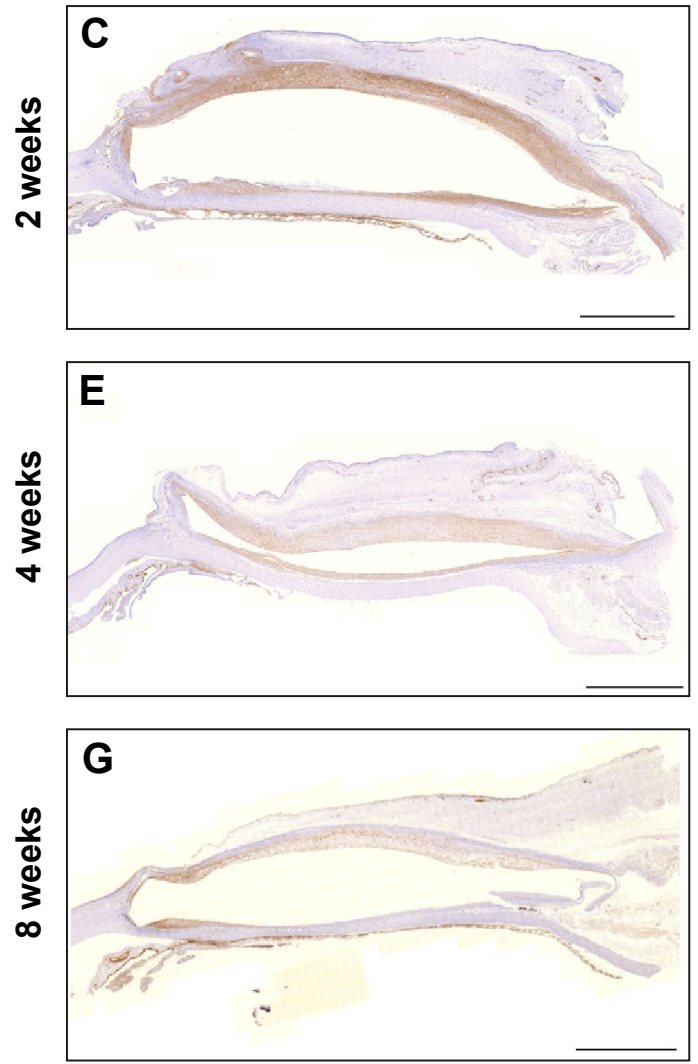

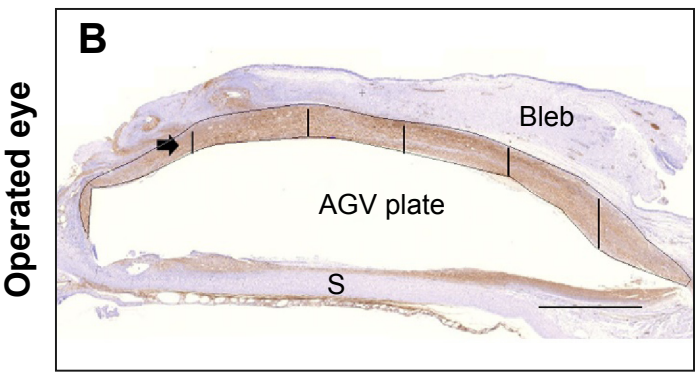

Pirfenidone
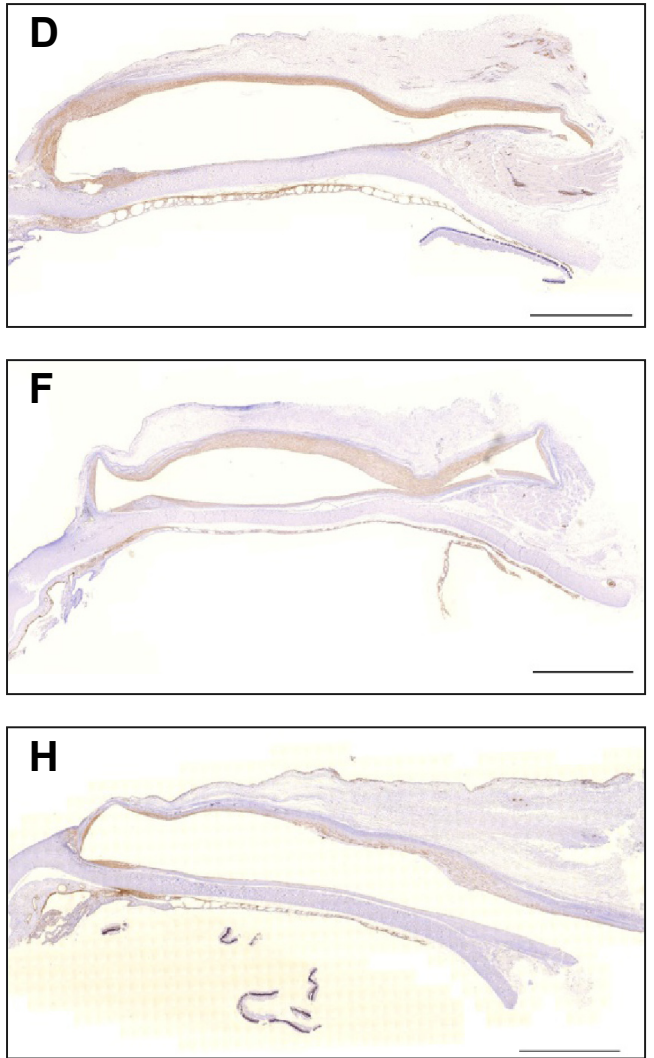
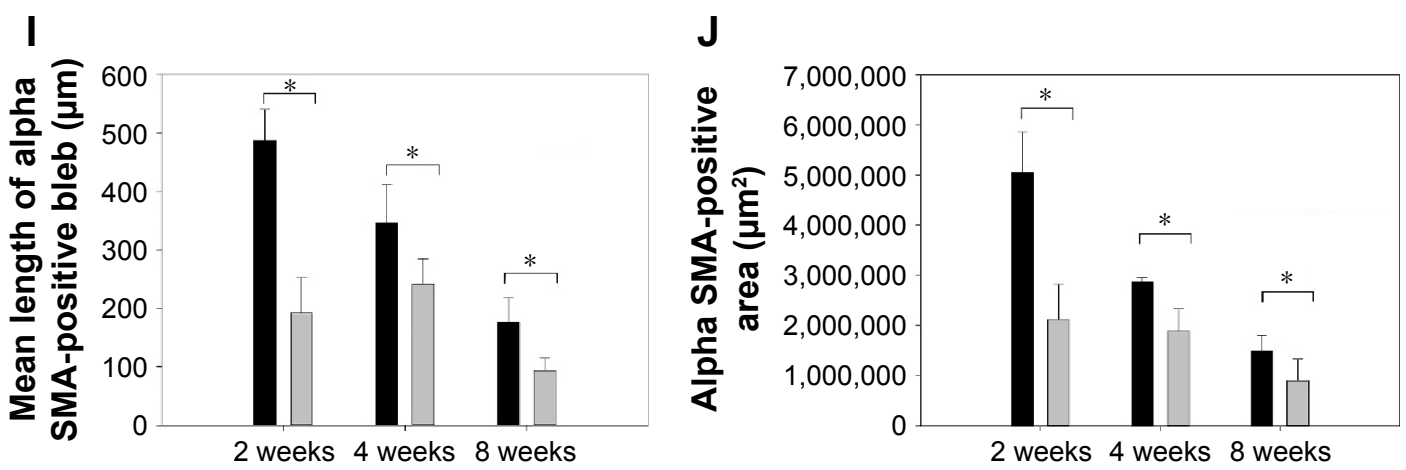

Control $\square$ Pirfenidone

Figure 6 Immunohistochemical micrographs for transition from fibroblasts to myofibroblasts.

Notes: No anti- $\alpha$-SMA immunostaining was visible in virgin conjunctiva and sclera (A). The mean of five height values (arrow) and the total area of the $\alpha$-SMA immunostained bleb were measured (B). Immunostaining for $\alpha$-SMA was greatest in the inner portion of the bleb from 2 weeks to 8 weeks post operation in the pirfenidone (D, F, $\mathbf{H})$ and control (C, E, G) groups. The height (I) and area (J) of $\alpha$-SMA expression were lower in the pirfenidone group compared to the control group at 8 weeks post operation (all $P<0.05)$. Scale bars: $500 \mu \mathrm{m}(\mathbf{A}) ; 2 \mathrm{~mm}(\mathbf{B}-\mathbf{H})$. *A significant difference with $P<0.05$ compared to control rats.

Abbreviations: AGV, Ahmed glaucoma valve; CE, conjunctival epithelium; S, sclera; SMA, smooth muscle actin; TC, Tenon's capsule. 
and both decreased by 8 weeks post operation. The height and area of $\alpha$-SMA expression were lower in the pirfenidone group than in the control group at 2 weeks, 4 weeks, and 8 weeks post operation (all $P<0.05$; height, Figure 6I; area, Figure 6J).

\section{Discussion}

In the rabbit model of GDD implantation, we demonstrated that pirfenidone decreased fibrosis, especially the thickness of the inner fibrotic layer in blebs. Proliferation of fibroblasts was significantly lower in the pirfenidone group than in the control group at 2 weeks post operation. Pirfenidone treatment suppressed expression of CTGF and the transformation of fibroblasts into myofibroblasts.

In glaucoma filtration surgeries, excessive postoperative scarring is the important determinant for bleb failure with inadequate IOP control..$^{23,24}$ After trabeculectomy failure, or in complicated glaucoma in which the rate of failure of simple filtration surgery is high, GDDs are often used to manage IOP. ${ }^{25}$ The most common long-term complication of seton glaucoma surgery is failure due to the formation of a thick fibrous capsule around the device. ${ }^{9,25}$ Control of IOP in implant surgery may rely on the bleb wall thickness and the total surface area of the bleb. ${ }^{7-9}$

The wound-healing response in GDD implantation differs from that in trabeculectomy because of the occurrence of foreign body reaction, which is characterized by the formation of a dense fibrous capsule with FBGCs. ${ }^{10}$ Nakamura-Shibasaki et al showed that at 10 days after surgery, in a rat model, the extent of scarring was greater following implant surgery than after filtration surgery. ${ }^{26}$ Anterior-segment optical coherence tomography imaging showed that, following implant surgery, blebs displayed a homogeneous and hyperreflective bleb wall, implying dense fibrotic capsule formation, whereas after trabeculectomy the blebs had multiple subconjunctival fluid collections. ${ }^{9,27}$ Although the introduction of mitomycin $\mathrm{C}$ has increased the success rate of trabeculectomy, adjunctive antimetabolites did not improve surgical outcomes following GDD implantation but were associated with similar or shorter survival rates and increased postoperative complications such as wound leakage or hypotony. ${ }^{28-31}$ Poor outcomes with mitomycin $\mathrm{C}$ may result from the specialty of foreign body reaction with formation of fibrotic capsule. There is a need for other potent therapeutic strategies to decrease the thickening of the tissues surrounding the endplate, especially because GDD failure remains a significant problem in refractory glaucoma.

In the present study, pirfenidone reduced collagen deposition in the bleb, especially in the innermost fibrotic layer at 4 weeks and 8 weeks post operation following GDD implantation. This finding is in agreement with many studies demonstrating attenuation of extracellular matrix deposition, including collagen, by pirfenidone treatment. ${ }^{32,33}$ Zhong et $\mathrm{al}^{34}$ reported that topical application of $0.5 \%$ pirfenidone elevated bleb survival after trabeculectomy in rabbits.

Although the precise mechanism of its action has not been determined, the antifibrotic effects of pirfenidone have relevance in the control of cytokines and proteins, the best known effect being attenuation of TGF- $\beta .{ }^{17,18}$ In an in vitro study using Tenon's fibroblasts, pirfenidone downregulated expression of TGF- $\beta 1,-\beta 2$, and $-\beta 3$. $^{21}$ There are a number of reasons why we should focus on the anti-TGF- $\beta$ activity of pirfenidone. One of the main modulators in the woundhealing process is TGF- $\beta$, which is secreted by platelets, macrophages, and fibroblasts. ${ }^{12}$ TGF- $\beta$ plays a critical role in inducing fibroblasts to synthesize collagen and transform into myofibroblasts. ${ }^{12}$ In glaucoma implant surgery, the insertion of a device into the subtenon space is indispensable. This results in a foreign body reaction, including the formation of FBGCs and a fibrotic capsule that surrounds the device. In such a reaction, TGF- $\beta$ is also a main mediator in the encapsulation of the device and fibrosis. ${ }^{35}$ Aqueous humor flows through the device and pools over the endplate, affecting the foreign body reaction in GDD surgery. ${ }^{10}$ Aqueous humor aggravates fibrosis following implant surgery, which might be induced by many growth factors. ${ }^{10}$ Among the many growth factors in aqueous humor, TGF- $\beta$ is predominant. ${ }^{36-38}$ Much of the TGF- $\beta$ (15\%-100\%, with a mean value of $61 \% \pm 27 \%$ ) in aqueous humor is present in its active form. ${ }^{37}$ Given the function of TGF- $\beta$ in the wound-healing response, including the foreign body reaction, the anti-TGF- $\beta$ activity of pirfenidone could attenuate fibrosis in the bleb following GDD surgery.

The TGF- $\beta$ downstream signaling molecule, CTGF, plays an important role in fibrosis and wound contraction. ${ }^{39}$ Blocking of CTGF suppresses TGF- $\beta$-induced fibroblast proliferation or collagen synthesis. ${ }^{40-42}$ In this study, we demonstrated that CTGF, a downstream effector of TGF- $\beta$, was expressed in bleb tissue following implant surgery. Pirfenidone treatment decreased the expression of CTGF in blebs at 2 weeks post operation. Several studies have reported that pirfenidone suppresses mRNA or protein expression of CTGF in cultured fibroblasts or in nephrectomized rats. ${ }^{19,43,44}$

Proliferation of fibroblasts as determined by PCNA immunoreactivity was weaker in the pirfenidone group than in the control group after GDD implantation. This observation is in concurrence with previous studies showing that pirfenidone decreased the proliferation of fibroblasts in an in vitro or in vivo trabeculectomy animal model. ${ }^{21,34}$ 
Pirfenidone had an inhibitory effect on the transition of fibroblasts to myofibroblasts at 2 weeks, 4 weeks, and 8 weeks post operation. Myofibroblasts are critical in the accumulation of extracellular matrix and wound contraction. ${ }^{22}$ TGF- $\beta$ is the main regulator in stimulating the transformation of fibroblasts to myofibroblasts. ${ }^{45}$ In GDD surgery, pirfenidone may block the expression of $\alpha$-SMA through anti-TGF- $\beta$ activity. Exposure to pirfenidone decreased $\alpha$-SMA levels in Tenon's fibroblasts in in vitro and in vivo studies, as in our findings. ${ }^{34,44,46,47}$

We chose to apply pirfenidone as one intrableb injection immediately after surgery with subsequent topical application. Intrableb injection of a drug may directly affect the wound site, but repeated injection can induce microtrauma to the bleb leading to unexpected bleeding. Therefore, we applied pirfenidone as eye drops after one local injection.

A small sample size per group $(n=5)$ was the limitation of this study. In this study, we found no significant difference in postoperative IOPs between the two groups. This might have been due to not using a glaucoma model with elevation of IOP. In the rabbit, the wound-healing response is more aggressive than in humans. Glaucoma filtration surgeries tend to fail earlier in rabbits than in glaucoma patients. ${ }^{48,49}$ Therefore, the effects of pirfenidone in GDD surgery in the rabbit may be reproducible clinically.

There have been no established definite standards for the evaluation of bleb fibrosis. Quantitative measurement of the inner bleb layer can be a standard for the evaluation of bleb fibrosis. Eight weeks post operation, the innermost layers that demonstrated a densely packed collagenous stratum were intensively stained blue by Masson trichrome stain. $\alpha$-SMA was stained exclusively in the inner bleb area. We suppose that the inner bleb layer is significant for resistance of aqueous humor flow through the bleb wall. Assessment of inner bleb wall also can make up for the weak points of analysis for the total bleb areas because the total bleb area can vary from section to section.

However, PCNA or CTGF expression was seen to a certain degree also in the outer bleb. Therefore, evaluation of total bleb area was also performed for PCNA or CTGF in addition to inner bleb area. The cross section just in the middle portion of the bleb was analyzed to decrease the subjectivity of image evaluation. We believe that consistent location in sections of the bleb is possible.

\section{Conclusion}

There has been little research on aqueous shunts and efforts to decrease the thickness of the fibrotic capsule, even though GDD failure remains a significant concern in complicated glaucoma. In conclusion, this study suggests that pirfenidone treatment can be considered as a strategy to decrease fibrotic encapsulation in foreign body reaction following GDD implantation.

\section{Acknowledgments}

This research was supported by Basic Science Research Program through the National Research Foundation of Korea (NRF) funded by the Ministry of Education (2014R1A1A2059143) and Research Fund of Seoul St. Mary's Hospital, The Catholic University of Korea. The Ahmed valves used in this study were supplied by New World Medical Inc. (Rancho Cucamonga, CA, USA). The authors declare that no competing interests exist with the commercial funder. The funders had no role in study design, data collection and analysis, decision to publish, or preparation of the manuscript.

\section{Disclosure}

The authors report no conflicts of interest in this work.

\section{References}

1. Almasieh M, Wilson AM, Morquette B, Cueva Vargas JL, Di Polo A. The molecular basis of retinal ganglion cell death in glaucoma. Prog Retin Eye Res. 2012;31(2):152-181.

2. Chen PP, Yamamoto T, Sawada A, Parrish RK 2nd, Kitazawa Y. Use of antifibrosis agents and glaucoma drainage devices in the American and Japanese Glaucoma Societies. J Glaucoma. 1997;6(3):192-196.

3. Joshi AB, Parrish RK 2nd, Feuer WF. 2002 survey of the American Glaucoma Society: practice preferences for glaucoma surgery and antifibrotic use. J Glaucoma. 2005;14(2):172-174.

4. Desai MA, Gedde SJ, Feuer WJ, Shi W, Chen PP, Parrish RK 2nd. Practice preferences for glaucoma surgery: a survey of the American Glaucoma Society in 2008. Ophthalmic Surg Lasers Imaging. 2011;42(3):202-208.

5. Gedde SJ, Schiffman JC, Feuer WJ, et al. Treatment outcomes in the tube versus trabeculectomy (TVT) study after five years of follow-up. Am J Ophthalmol. 2012;153(5):789e2-803e2.

6. Smith SL, Starita RJ, Fellman RL, Lynn JR. Early clinical experience with the Baerveldt 350-mm2 glaucoma implant and associated extraocular muscle imbalance. Ophthalmology. 1993;100(6):914-918.

7. Schwartz KS, Lee RK, Gedde SJ. Glaucoma drainage implants: a critical comparison of types. Curr Opin Ophthalmol. 2006;17(2):181-189.

8. Heuer DK, Lloyd MA, Abrams DA, et al. Which is better? One or two? A randomized clinical trial of single-plate versus double-plate Molteno implantation for glaucomas in aphakia and pseudophakia. Ophthalmology. 1992;99(10):1512-1519.

9. Jung KI, Lim SA, Park HY, Park CK. Visualization of blebs using anterior-segment optical coherence tomography after glaucoma drainage implant surgery. Ophthalmology. 2013;120(5):978-983.

10. Jung KI, Lee SB, Kim JH, Park CK. Foreign body reaction in glaucoma drainage implant surgery. Invest Ophthalmol Vis Sci. 2013;54(6): 3957-3964.

11. Welge-Lüssen U, May CA, Neubauer AS, Priglinger S. Role of tissue growth factors in aqueous humor homeostasis. Curr Opin Ophthalmol. 2001;12(2):94-99.

12. Teller P, White TK. The physiology of wound healing: injury through maturation. Surg Clin North Am. 2009;89(3):599-610.

13. Iyer SN, Wild JS, Schiedt MJ, Hyde DM, Margolin SB, Giri SN. Dietary intake of pirfenidone ameliorates bleomycin-induced lung fibrosis in hamsters. J Lab Clin Med. 1995;125(6):779-785. 
14. Iyer SN, Gurujeyalakshmi G, Giri SN. Effects of pirfenidone on transforming growth factor-beta gene expression at the transcriptional level in bleomycin hamster model of lung fibrosis. J Pharmacol Exp Ther. 1999;291(1):367-373.

15. du Bois RM. Strategies for treating idiopathic pulmonary fibrosis. Nat Rev Drug Discov. 2010;9(2):129-140.

16. Raghu G, Collard HR, Egan JJ, et al. An official ATS/ERS/JRS/ALAT statement: idiopathic pulmonary fibrosis: evidence-based guidelines for diagnosis and management. Am J Respir Crit Care Med. 2011; 183(6):788-824.

17. Schaefer CJ, Ruhrmund DW, Pan L, Seiwert SD, Kossen K. Antifibrotic activities of pirfenidone in animal models. Eur Respir Rev. 2011; 20(120):85-97.

18. Richeldi L, Yasothan U, Kirkpatrick P. Pirfenidone. Nat Rev Drug Discov. 2011;10(7):489-490.

19. Hewitson TD, Kelynack KJ, Tait MG, et al. Pirfenidone reduces in vitro rat renal fibroblast activation and mitogenesis. J Nephrol. 2001; 14(6):453-460.

20. Gurujeyalakshmi G, Hollinger MA, Giri SN. Pirfenidone inhibits PDGF isoforms in bleomycin hamster model of lung fibrosis at the translational level. Am J Physiol. 1999;276(2 Pt 1):L311-L318.

21. Lin X, Yu M, Wu K, Yuan H, Zhong H. Effects of pirfenidone on proliferation, migration, and collagen contraction of human Tenon's fibroblasts in vitro. Invest Ophthalmol Vis Sci. 2009;50(8):3763-3770.

22. Tomasek JJ, Gabbiani G, Hinz B, Chaponnier C, Brown RA. Myofibroblasts and mechano-regulation of connective tissue remodelling. Nat Rev Mol Cell Biol. 2002;3(5):349-363.

23. Hitchings RA, Grierson I. Clinico pathological correlation in eyes with failed fistulizing surgery. Trans Ophthalmol Soc U K. 1983;103(Pt 1): 84-88.

24. Addicks EM, Quigley HA, Green WR, Robin AL. Histologic characteristics of filtering blebs in glaucomatous eyes. Arch Ophthalmol. 1983;101(5):795-798.

25. Jacob JT, Burgoyne CF, McKinnon SJ, Tanji TM, LaFleur PK, Duzman E. Biocompatibility response to modified Baerveldt glaucoma drains. J Biomed Mater Res. 1998;43(2):99-107.

26. Nakamura-Shibasaki M, Ko JA, Takenaka J, Chikama T, Sonoda KH, Kiuchi Y. Matrix metalloproteinase and cytokine expression in Tenon fibroblasts during scar formation after glaucoma filtration or implant surgery in rats. Cell Biochem Funct. 2013;31(6):482-488.

27. Thieme H, Choritz L, Hofmann-Rummelt C, Schloetzer-Schrehardt U, Kottler UB. Histopathologic findings in early encapsulated blebs of young patients treated with the ahmed glaucoma valve. J Glaucoma. 2011;20(4):246-251.

28. Prata JA Jr, Minckler DS, Mermoud A, Baerveldt G. Effects of intraoperative mitomycin-C on the function of Baerveldt glaucoma drainage implants in rabbits. J Glaucoma. 1996;5(1):29-38.

29. Lee D, Shin DH, Birt CM, et al. The effect of adjunctive mitomycin C in Molteno implant surgery. Ophthalmology. 1997;104(12): 2126-2135.

30. Costa VP, Azuara-Blanco A, Netland PA, Lesk MR, Arcieri ES. Efficacy and safety of adjunctive mitomycin C during Ahmed Glaucoma Valve implantation: a prospective randomized clinical trial. Ophthalmology. 2004;111(6):1071-1076.

31. Al-Mobarak F, Khan AO. Two-year survival of Ahmed valve implantation in the first 2 years of life with and without intraoperative mitomycin-C. Ophthalmology. 2009;116(10):1862-1865.

Drug Design, Development and Therapy

\section{Publish your work in this journal}

Drug Design, Development and Therapy is an international, peerreviewed open-access journal that spans the spectrum of drug design and development through to clinical applications. Clinical outcomes, patient safety, and programs for the development and effective, safe, and sustained use of medicines are a feature of the journal, which
32. Kim H, Choi YH, Park SJ, et al. Antifibrotic effect of pirfenidone on orbital fibroblasts of patients with thyroid-associated ophthalmopathy by decreasing TIMP-1 and collagen levels. Invest Ophthalmol Vis Sci. 2010;51(6):3061-3066.

33. Choi K, Lee K, Ryu SW, Im M, Kook KH, Choi C. Pirfenidone inhibits transforming growth factor-beta1-induced fibrogenesis by blocking nuclear translocation of Smads in human retinal pigment epithelial cell line ARPE-19. Mol Vis. 2012;18:1010-1020.

34. Zhong H, Sun G, Lin X, Wu K, Yu M. Evaluation of pirfenidone as a new postoperative antiscarring agent in experimental glaucoma surgery. Invest Ophthalmol Vis Sci. 2011;52(6):3136-3142.

35. Luttikhuizen DT, Harmsen MC, Van Luyn MJ. Cellular and molecular dynamics in the foreign body reaction. Tissue Eng. 2006; 12(7):1955-1970.

36. Granstein RD, Staszewski R, Knisely TL, et al. Aqueous humor contains transforming growth factor-beta and a small (less than 3500 daltons) inhibitor of thymocyte proliferation. J Immunol. 1990;144(8): 3021-3027.

37. Jampel HD, Roche N, Stark WJ, Roberts AB. Transforming growth factorbeta in human aqueous humor. Curr Eye Res. 1990;9(10):963-969.

38. Cousins SW, McCabe MM, Danielpour D, Streilein JW. Identification of transforming growth factor-beta as an immunosuppressive factor in aqueous humor. Invest Ophthalmol Vis Sci. 1991;32(8):2201-2211.

39. Schmidt W, Kastner C, Sternberg K, et al. New concepts for glaucoma implants - controlled aqueous humor drainage, encapsulation prevention and local drug delivery. Curr Pharm Biotechnol. 2013;14(1):98-111.

40. Kothapalli D, Frazier KS, Welply A, Segarini PR, Grotendorst GR. Transforming growth factor beta induces anchorage-independent growth of NRK fibroblasts via a connective tissue growth factor-dependent signaling pathway. Cell Growth Differ. 1997;8(1):61-68.

41. Kothapalli D, Hayashi N, Grotendorst GR. Inhibition of TGF-betastimulated CTGF gene expression and anchorage-independent growth by cAMP identifies a CTGF-dependent restriction point in the cell cycle. FASEB J. 1998;12(12):1151-1161.

42. Duncan MR, Frazier KS, Abramson S, et al. Connective tissue growth factor mediates transforming growth factor beta-induced collagen synthesis: down-regulation by cAMP. FASEB J. 1999;13(13):1774-1786.

43. Saito M, Yamazaki M, Maeda T, Matsumura H, Setoguchi Y, Tsuboi R. Pirfenidone suppresses keloid fibroblast-embedded collagen gel contraction. Arch Dermatol Res. 2012;304(3):217-222.

44. Chen JF, Ni HF, Pan MM, et al. Pirfenidone inhibits macrophage infiltration in 5/6 nephrectomized rats. Am J Physiol Renal Physiol. 2013; 304(6):F676-F685.

45. Biernacka A, Dobaczewski M, Frangogiannis NG. TGF-beta signaling in fibrosis. Growth Factors. 2011;29(5):196-202.

46. Na JH, Sung KR, Shin JA, Moon JI. Antifibrotic effects of pirfenidone on Tenon's fibroblasts in glaucomatous eyes: comparison with mitomycin C and 5-fluorouracil. Graefes Arch Clin Exp Ophthalmol. 2015;253(9):1537-1545.

47. Jung KI, Choi JS, Kim HK, Shin SY. Effects of an anti-transforming growth factor-beta agent (pirfenidone) on strabismus surgery in rabbits. Curr Eye Res. 2012;37(9):770-776.

48. Seetner A, Morin JD. Healing of trabeculectomies in rabbits. Can J Ophthalmol. 1979;14(2):121-125.

49. Schwade ND, Chiou GC. The use of trabeculectomy to study the pharmacological modulation of ophthalmic wound healing. $J$ Ocul Pharmacol Ther. 1995;11(2):113-123.

\section{Dovepress}

has also been accepted for indexing on PubMed Central. The manuscript management system is completely online and includes a very quick and fair peer-review system, which is all easy to use. Visit http://www.dovepress.com/testimonials.php to read real quotes from published authors.

Submit your manuscript here: http://www.dovepress.com/drug-design-development-and-therapy-journal 